\title{
Influence of the Limited Retransmission on the Performance of WLANs Using Error-Prone Channel
}

\author{
Haider M. ALSABBAGH ${ }^{1}$, Jianping CHEN, Youyun XU \\ Department of Electronic Engineering, Shanghai Jiao Tong University, Shanghai, P.R.China \\ E-mail: ${ }^{1}$ haidermaw@gmail.com
}

\begin{abstract}
In WLANs, stations sharing a common wireless channel are governed by IEEE 802.11 protocol. Many conscious studies have been conducted to utilize this precious medium efficiently. However, most of these studies have been done either under assumption of idealistic channel condition or with unlimited retransmitting number. This paper is devoted to investigate influence of limited retransmissions and error level in the utilizing channel on the network throughput, probability of packet dropping and time to drop a packet. The results show that for networks using basic access mechanism the throughput is suppressed with increasing amount of errors in the transmitting channel over all the range of the retry limit. It is also quite sensitive to the size of the network. On the other side, the networks using four-way handshaking mechanism has a good immunity against the error over the available range of retry limits. Also the throughput is unchangeable with size of the network over the range of retransmission limits. However, the throughput does not change with retry limits when it exceeds the maximum number of the backoff stage in both DCF's mechanisms. In both mechanisms the probability of dropping a packet is a decreasing function with number of retransmissions and the time to drop a packet in the queue of a station is a strong function to the number of retry limit, size of the network, the utilizing medium access mechanism and amount of errors in the channel.
\end{abstract}

Keywords: IEEE802.11 DCF, WLAN, MAC Protocol, Throughput, Error-prone Channel.

\section{Introduction}

Next generation communications networks are being investigated thoroughly to satisfy its ultimate goal: accessible at any time and anywhere. One key point to satisfy such aim is to increase both data rate and processing speed [1][2]. The Wireless local area networks (WLANs) are able to comply with such demands and have become one of the fastest growing segments in the communications industry, especially with utilizing the new version of its protocol IEEE 802.11n [3]. The worldwide shipments of the WLAN equipment products reach $\$ 5.9$ billion in 2006, and it is expected that WLAN equipment will continue to grow in 2007 to reach around $\$ 6.5$ billion level as new IEEE $802.11 \mathrm{n}$ and VoWi-Fi equipment is introduced and the infrastructure for traditional Wi-Fi expands [3][4]. In 1997 IEEE's committee standardized 802.11 protocol for WLANs [5]. Since that time several versions of this protocol have been made. The physical media in the WLANs is shared between all stations and has limited connection range compared with its wired counterpart. The standard defines three PHY technologies and a unified MAC protocol to support 1 and 2 Mbps transmission over wireless media.
The MAC protocol has two functions, namely distributed coordination function (DCF) and the optional point coordination function (PCF). DCF has superior attractiveness over PCF in many aspects [6], therefore this study is conducted to investigate WLANs utilizing DCF. DCF defines two mechanisms to access transmission medium: the basic access scheme, which is the default scheme and the request to send/clear to send (RTS/CTS) scheme, also known as four-way handshaking scheme [4][7][8]. Recently, considerable studies have been concentrated on modeling the IEEE 802.11 DCF medium access method. Bianchi in [9] modeled the idealistic assumption of collision only errors and packet retransmits are unlimited; a packet is being transmitted continuously until its successful reception. $\mathrm{Wu}$ in [10] extended Bianchi's analysis to include the finite packet retry limits as defined in the standard. Both studies used Markov chain model to analyze DCF operation and calculated the saturated throughput of 802.11 protocol. Periklais et al. [11] extended the work in [9] and [10] by taking into account both: transmission errors and packet retry limits for basic access of the IEEE802.11a protocol. $\mathrm{X}$. Wang et al. [12] evaluate the impact of transmission error rate on the contention and the system throughput in 
WLAN's protocol. However, [11] and [12] considered the probability of bit errors appearing on the transmission channel is the same in the two access mechanisms. $Z$. Tang et al. [13] presented an analytical model to evaluate the performance of the DCF in the case of bit errors appearing on the transmission channel and taking type of the used mechanism into account. However, Tang's study was under assuming of unlimited retransmitting. In this paper we extend Tang's work by taking into account influence of retransmissions and investigate its impacts on the performance of the WLANs. The results show that the throughput is insensitive to the number of the retransmissions when it exceeds the maximum number of backoff stages in both mechanisms. This insensitive trend does not change with amount of BER in the utilizing channel. However, adopting four-way handshaking mechanism show that the throughput is more immunes than its counterpart mechanism when WLAN's channel suffers from much error. This paper is organized as follows: Section 2 devoted to explain the used model in this study. Explanations for the achieved results are given in Section 3 and then our conclusions are drawn to Section 4.

\section{The Model}

The analysis employs the Markov chain model in [8] and [10] and makes use of the same assumptions as in [13]: all stations always have a packet available for transmitting (saturation case) into an error prone-channel.

\subsection{A Brief Description of the Backoff Process in IEEE 802.11 DCF}

When stations sense that the medium is idle for a period, more than DCF, the backoff timer value for each station is uniformly chosen within the interval $\left[0, W_{i}-1\right]$, where $W_{i}$ is the current contention window $(\mathrm{cw})$ size and $i$ is the backoff stage $i \in[0, m]$ and $m$ represents the station's retry limit. The backoff counter for every station depends on the collision and on the successful packet transmissions experienced by the station in the past. At the beginning: $W=W_{o}$ and after each retransmitting due to a packet collision or error, $W_{i}$ is doubled up to a maximum value, ${ }^{W_{m}^{\prime}}=2^{m^{\prime}} W$, where $m^{\prime}$ is the maximum number of the backoff stages. When $W_{i}$ reaches $W_{m}$, it will stay at this value until it is reset to $W_{o}$ again either after the successful data the counter reaches to its limit.

\subsection{The analytical modeling ${ }^{1}$}

As in [10], the probability of a station to transmit a packet in a randomly chosen slot time is:

\footnotetext{
${ }^{1}$ Definition and values of all the rest parameters that do no mentioned here are as in Ref. [13].
}

$$
\tau=\frac{1-p^{m+1}}{(1-p)} \cdot \Psi_{0,0}
$$

where:

$$
\Psi_{0,0}= \begin{cases}\frac{2(1-2 p) \cdot(1-p)}{W\left(1-(2 p)^{m+1} \cdot(1-p)+(1-2 p) \cdot\left(1-p^{m+1}\right)\right)} & m \leq m^{\prime} \\ \frac{2(1-2 p) \cdot(1-p)}{W\left(1-(2 p)^{m^{\prime}+1}\right) \cdot(1-p)+(1-2 p) \cdot\left(1-p^{m+1}\right)+W \cdot 2^{m^{\prime}} p^{m^{\prime}+1} \cdot(1-2 p) \cdot\left(1-p^{m-m^{\prime}}\right)} m>m^{\prime}\end{cases}
$$

$\tau$ does not depend on the type of the mechanism adapted by a station: basic access or four-way handshaking, $P$ is the unsuccessful probability when a transmitted packet encounters a collision with at least one of the $n-1$ remaining stations in a time slot. So:

$$
p=1-(1-\tau)^{n-1}\left(1-p_{c}\right)
$$

Influence of errors in the transmitting channel is included through the parameter $\boldsymbol{P}_{\boldsymbol{C}}$ as [13]: In the case of basic access mechanism:

$$
p_{c}=1-(1-B E R)^{L}
$$

where $L=P H Y_{h}+M A C_{h}+P+A C K$. In the case of four-way handshaking:

$$
P_{C}=1-(1-B E R)^{R T S} *_{(1-B E R)} C T S *_{*(1-B E R)^{P}(1-B E R)} A C K
$$

When a station transmits and the remaining $\boldsymbol{n - 1}$ stations defer their transmissions, the packet would be arriving successfully with probability $\boldsymbol{P}_{\boldsymbol{S}}$. Considering the probability that a random slot is empty $\left(\mathbf{1 - P _ { t r } )}\right.$, probability of successful transmission is $\boldsymbol{P}_{t r} \boldsymbol{P}_{\boldsymbol{s}}$ and probability of the collision is $\boldsymbol{P}_{t r}\left(\mathbf{1 - P _ { S }}\right)$, the average length of a slot time is:

$$
E[s l o t]=\left(1-p_{t r}\right) \cdot \sigma+p_{t r} \cdot p_{s} \cdot T_{s}+p_{t r} \cdot\left(1-p_{s}\right) \cdot T_{c}
$$

Consequently, the system throughput, can be expressed by dividing the successfully transmitted payload data over a slot time. The probability of dropping a packet when the retry limit is reached is known as the packet drop probability and given as:

$$
p_{\text {drop }}=p^{m+1}
$$

A packet is dropped when it reaches the last backoff stage and experiences another collision or an error.

\section{Performance Analysis}

\subsection{Influence of the Retry Limits on the Network Characteristics}

This analysis is based on the model in [13] and we have included influence of limited number of retransmissions. To validate our evaluation and highlight influence of limited retries we compare our results, after taking influence of limited retries, with results that have been gotten by using the model in [12] and with that using model given in Ref. [13]. The results are illustrated in Figure 1. The same parameters values in [13] have 
been used in this study in order to facilitate the comparison purpose. In Figure 1 our results denoted as (a), results of Ref. [13] denoted as (b) and the results that obtained by using the model in [12] denoted as (c). The system throughput has been estimated for three different network sizes: 5 (small), 20 (middle), and 50 (large). Figure 1 shows that: for networks utilizing the basic access, results (a) are much closer to results (b) in the small and middle network sizes and much close to results (c) for the large networks over all the range of BERs. This can be justified since on the small and middle networks the rate of collisions is relatively small compared with that in large networks, which indicates that influence of retransmission at small and middle networks is also small. For networks utilizing the four-way handshaking, all results: (a), (b) and (c) show that the throughputs are insensitive to size of the networks over all the examined range of the BERs. While almost all the results are mostly closed when BERs less than $10^{-5}$, there is a difference between the results (a) and (b) in one side and results (c) on the other side when $10^{-5}<B E R<10^{-4}$. This difference is due to the effect of the parameter $\boldsymbol{P}_{\boldsymbol{C}}$ on $\boldsymbol{P}$, which could be much sensible at higher level of BERs. Considering errors in the channel, $\boldsymbol{P}_{\boldsymbol{C}}$ in the Ref. [12] did not distinguish the used access mechanism as it did in [13] and in ours as shown in Eq. 3.

Figure 2 illustrates variation of the network throughput with number of retransmissions at low level of BERs $=$ $10^{-5}$ (Figure 2a) and at relatively high level of BERs $10^{-4}$ (Figure 2b). It is obvious that trend of the network throughput is almost unchangeable with level of errors in the channel when a network uses the four-way handshaking scheme. In the contrary side, when the networks use basic access, the throughput level is obviously decreased with increasing errors in the channel over all the retransmission range. Figure 3 shows that probability to drop a packet is exponentially decreasing with number of retransmissions and has undistinguishable differences between the two access mechanisms. However, this probability is increasing function of errors in the channel. Probability of dropping a packet is ignorable when the network exceeds the maximum number of backoff stage ${ }^{2}$ with low level of BER (see Figure 3 a). This leads to enforce packets stay at queue of the transmitter for a long time and cause much delay, which could be unacceptable for some delay-sensitive applications. Furthermore, Figure $4 \mathrm{a}$ and $\mathrm{b}$ show that the average time to drop a packet when adapting four-way handshaking is lower than that when using basic access at low level of BER and it becomes more obvious at high level of BER (as in Figure 4-b). These are beneficial of using RTS/CTS packets by reserving the channel in

\footnotetext{
${ }^{2}$ Extensive calculations for different values have been done, and all the obtained results were valid and assist the result stated in this paper: Probability of dropping a packet is ignorable when the network exceeds the maximum number of backoff stage.
}

advance before starting to transmit a long data packet and hence supporting reduction the rate of collisions.

\subsection{Network Characteristics with Specific Number of Retransmissions}

In this section, the model that developed in the section 2 will be used to investigate some characteristics of WLANs. IEEE $802.11 \mathrm{~b}$ is considered with retry limits $=5$. Figure 5 shows packet delay with channel capacity $C=1$, 5.5 and $11 \mathrm{Mbps}$ against the number of contending stations. Results indicate that packet delay increases linearly with increasing number of stations due to increase rate of collisions and hence increasing number of retry, which also causes increase of packet drop probability as clarified in Figure 6. However, Figure 7 shows that as transmission rate increases the packet drop time decreases remarkably. This is due to the time spent for packet transmission decreases as the data rate increases [11]. For large network sizes, the probability of collision increases and hence number of retransmitting increases for each sharing station. As a result, a station to get a chance to establish the new transmitting at large networks is lower than that in the small networks. Thus, the time to drop a packet in small networks is much suppressed, compared with that in large networks. For instance, for an access point in WLAN covering an area of 50 contending stations, the drop time is $7.57 \mathrm{~s}$ at $1 \mathrm{Mbps}$ bit rate and is $1.12 \mathrm{~s}$ at $11 \mathrm{Mbps}$ bit rate. Thus, from Figure 5, the corresponding packet delays are 573 $\mathrm{ms}$ and $85 \mathrm{~ms}$, respectively. Figures 8 and 9 indicate that using long packet payload leads to large packet delay and packet drop times. This is due to the collision time, $\boldsymbol{T}_{\boldsymbol{C}}$ which is proportional to the packet size as: $T_{C}=L+D I F S+\delta$, where $\mathrm{L}=(\mathrm{PHY}$ header + MAC header $)+$ packet payload, and $\delta$ is the propagation delay. The collision time is defined as the average time the channel is sensed busy by the stations during a collision. On the other side, the delay of a successful packet depends on the average number of slots required for a packet to experience $m+1$ collisions in the $(0,1, \ldots \ldots, m)$ stages and also depends on average slots time, which is proportional to the number of contending stations, and channel bit rates. Results in figures 8 and 9 investigate three different WLAN sizes: 5, 40, and 70 with three different channel bit rates: $1,5.5$ and $11 \mathrm{Mbps}$, the packet delay and packet drop time is linearly increasing with size of payload.

\section{Conclusion}

In this paper, an analytical model is developed to include influence of limited number of retransmissions on the main characteristics of WLANs that use error-prone channels. The model has been applied on the two available mechanisms in the DCF functions. We validate our evaluations by comparing our results with the results in two considered references. This study shows that: the networks using four-way handshaking mechanism have a 
good immunity against the increasing error in the transmitting channel over the range of retransmissions, also WLAN's throughput is unchangeable with size of the network over the range. On the other side, for the networks using basic access mechanism, the throughput is suppressed with increasing amount of errors in the transmission channel over all the available range of the retry limits as well as it is sensitive to the size of the network. Further, the throughput does not change with retry limits when it exceeds the maximum number of backoff stages in both DCF's mechanisms. In the both mechanisms probability of dropping a packet is decreasing function with number of retransmissions and the time to drop a packet in the queue of a station is strong function to the number of retry limits, size of the network, the utilized mechanism and the amount of errors in the transmitting channel. Also, the study investigates characteristics of the IEEE $802.11 \mathrm{~b}$ with a specific number of retry limits. The achieved results indicate that packet delay increases linearly with increasing number of stations due to increasing rate of collisions and hence increasing number of retry, which also causes increase of packet drop probability. Also, the network performance is strongly dependent on channel bit rate and the used packet length with adapting specific number of retransmission.

\section{References}

[1] G.-i. Ohta, F. Kamada, N. Teramura, and H. Hojo, " $5 \mathrm{GHz}$ W-LAN verification for public mobile applications -internet newspaper on train and advanced ambulance car," First IEEE Consumer Communications and Networking Conference. CCNC 2004, 2004, pp. 569-575.

[2] T. B. Zahariadis, K. G. Vaxevanakis, C. P. Tsantilas, and N. A. Zervos, "Global Roaming in Next-Generation Networks," IEEE Communications Magazine, February 2002, pp. 145-151.

[3] www.bbwexchange.com/publications/page1421-466 6.asp

[4] M. Etoh, "Next Generation Mobile Systems 3G and Beyond," John Wiley \& Sons, Ltd, 2005.
[5] "IEEE Standard for Wireless LAN Medium Access Control (MAC) and Physical Layer (PHY) Specifications ,ISO/IEC,” 1999, pp. 8802-11.

[6] H. Zhu and I. Chlamtac, "An Analytical Model for IEEE 802.11e EDCF Differential Services," The 12th international conference on Computer comunications and networks, ICCCN 2003, Preceedings, 2003, pp. 163-168.

[7] Y. F. Y. Kwon, and H. Latchman, "Design of MAC Protocols with Fast Collision Resolution for Wireless Local Area Networks," IEEE Transactions on wireless communications, vol. 3, MAY 2004, pp. 793-807.

[8] A. C. B. P. Chatzimisios, and V. Vitsas, "Performance analysis of the IEEE 802.11 MAC protocol for wireless LANs," Int. J. Commun.Syst., vol. 2, 2005, pp. 545-569.

[9] G.Bianchi, "Performance Analysis of the IEEE 802.11 Distributed Coordination Function," IEEE Journal on Selected Area in Communications, vol. 18, 2000, pp. 535-547.

[10] H. Wu, Y. Peng, K. Long, and J. Ma, "Performance of Reliable Transport Protocol over IEEE 802.11 Wireless LAN: Analysis and Enhancement," Proc. of IEEE INFOCOM, vol. 2, 2002, pp. 599-607.

[11] P. Chatzimisios, A. C. Boucouvalas, and V. Vitsas, "Performance Analysis of IEEE 802.11 DCF in Presence of Transmission Errors," IEEE International Conference on Communications, 2004, pp. 2854-2858.

[12] X. Wang, J. Yin, and D. P. Agrawal, "Impact of channel conditions on the throughput optimization in 802.11 DCF," Wirel. Commun. Mob. Comput., vol. 5, 2005, pp. 113-122.

[13] Z. Tang, Z. Yang, J. He, and YanweiLiu, "Impact of bit error on the performance of DCF for wireless LAN," Communications, Circuits and Systems and West Sino Expositions, IEEE 2002 International Conference, 2002, pp. 529-533. 


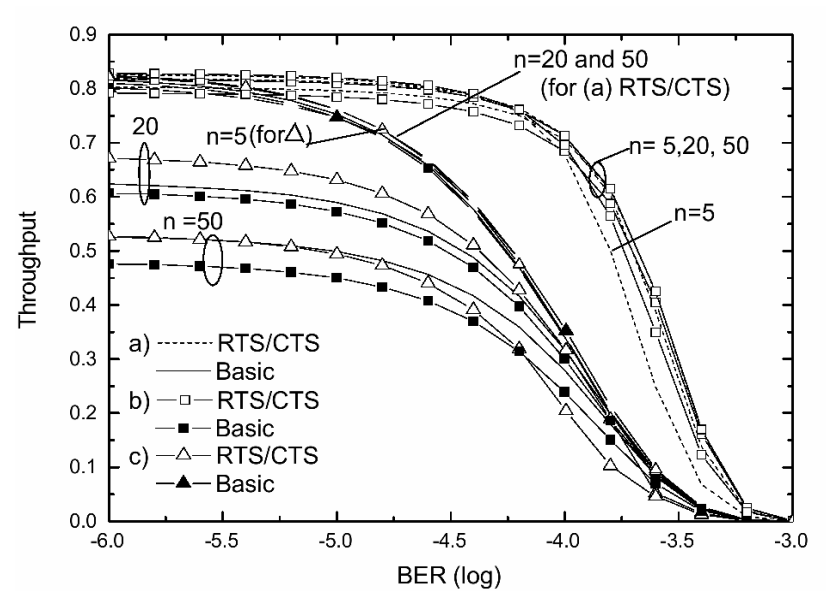

Figure 1. Throughput comparison for different network size $(n=5,20$ and 50): (a) our results, (b) results from Ref. [13], and (c) results obtained using the model in Ref.[12]

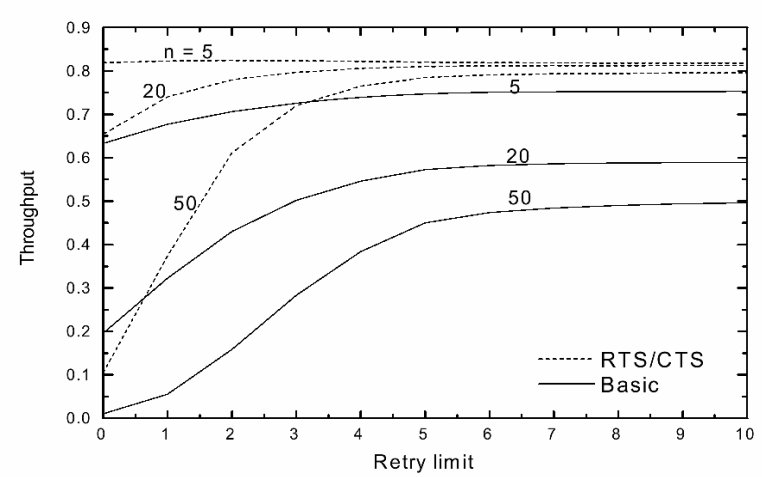

(a) $\mathrm{BER}=10^{-5}$

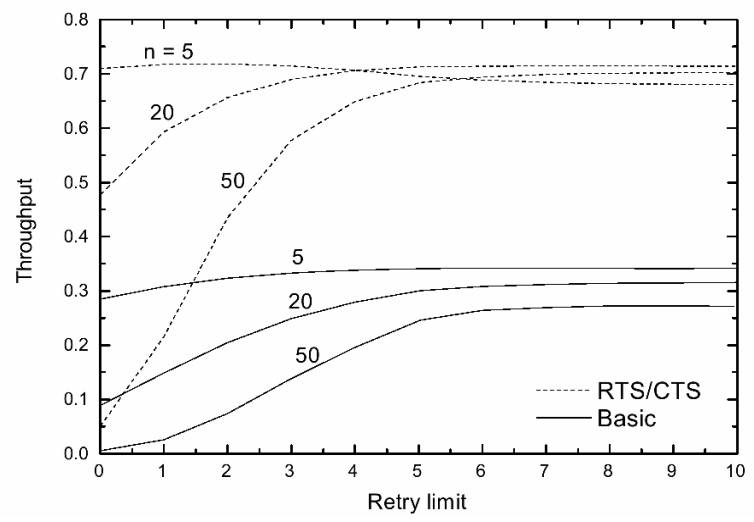

(b) $\mathrm{BER}=10^{-4}$

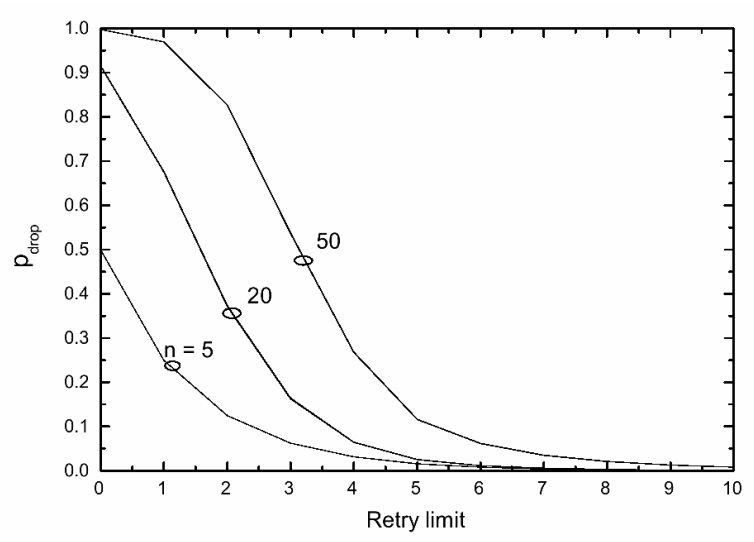

(a) $\operatorname{BER}=10^{-5}$

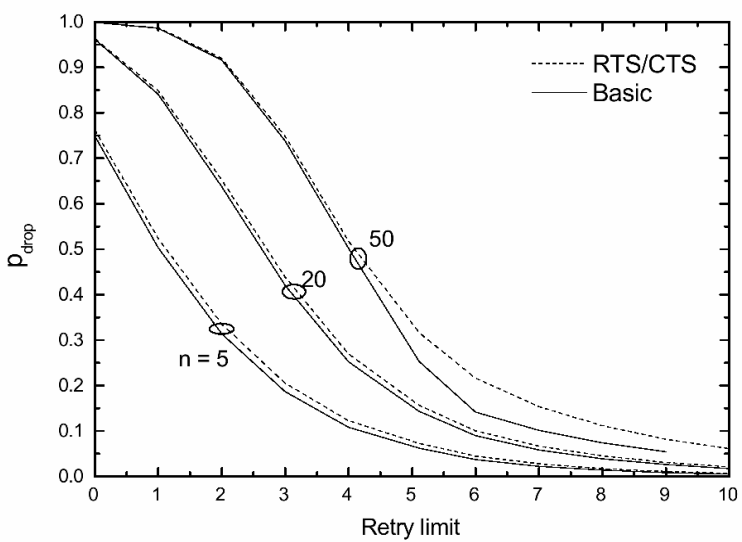

(b) $\mathrm{BER}=10^{-4}$

Figure 3. Impact of retry limit on the drop probability for different network sizes

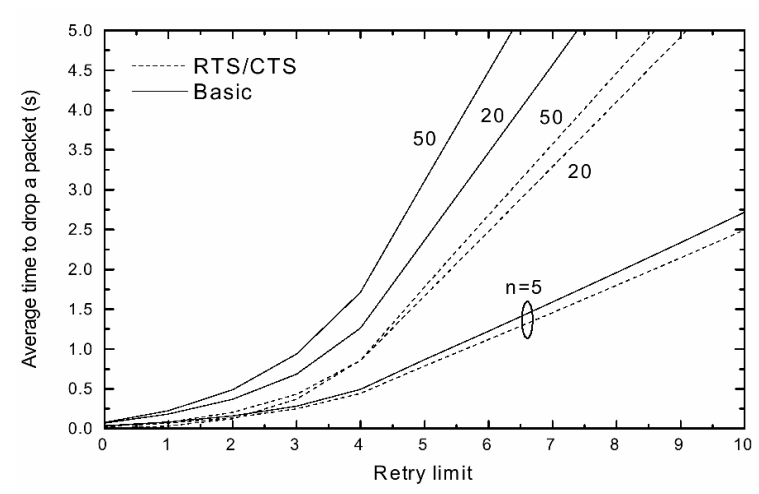

(a) $\mathrm{BER}=10^{-5}$

Figure 2. Throughput efficiency of the two mechanisms as a function of retry limit for different network sizes 


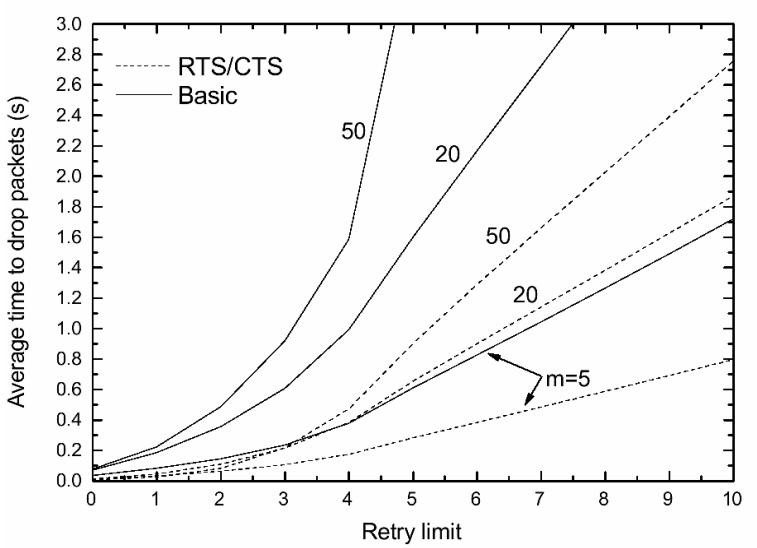

(b) $\mathrm{BER}=10^{-4}$

Figure 4. Influence of retry limit on the time to drop a packet for different network sizes

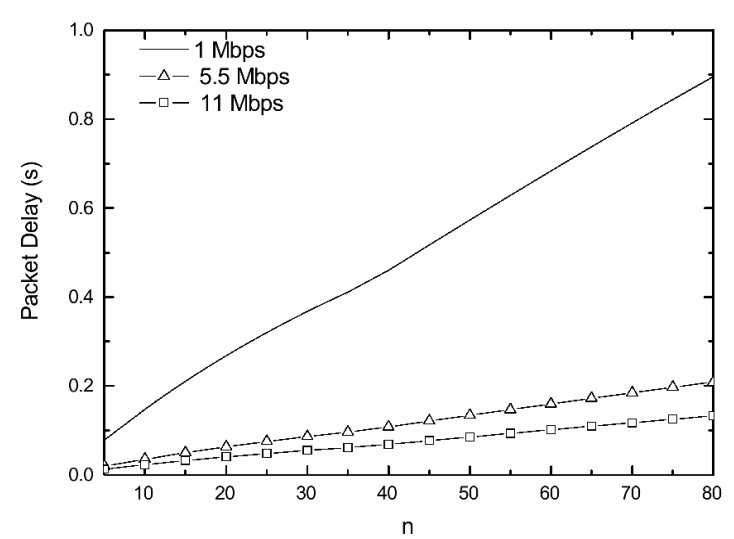

Figure 5. Packet delay with different channel capacities as a function of number of contending stations.

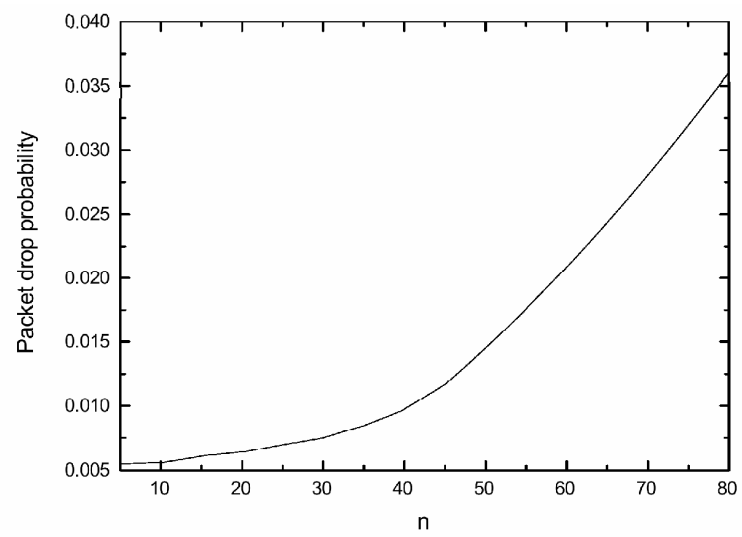

Figure 6. Packet drop probability against number of contending stations.

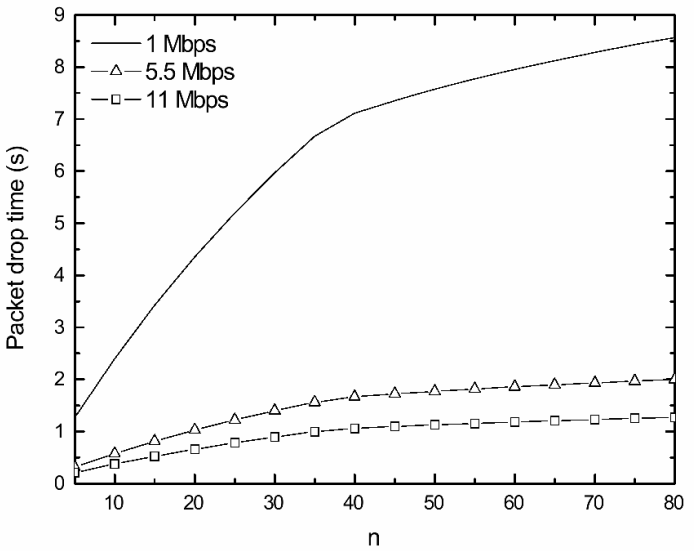

Figure 7. Packet drop time with different channel bit rates versus number of contending stations.

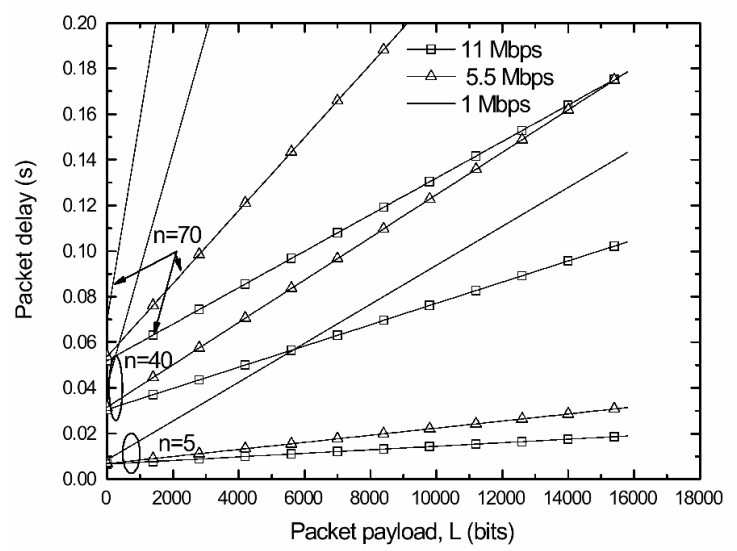

Figure 8. Packet delay against packet payload size for different contending stations and channel bit rates.

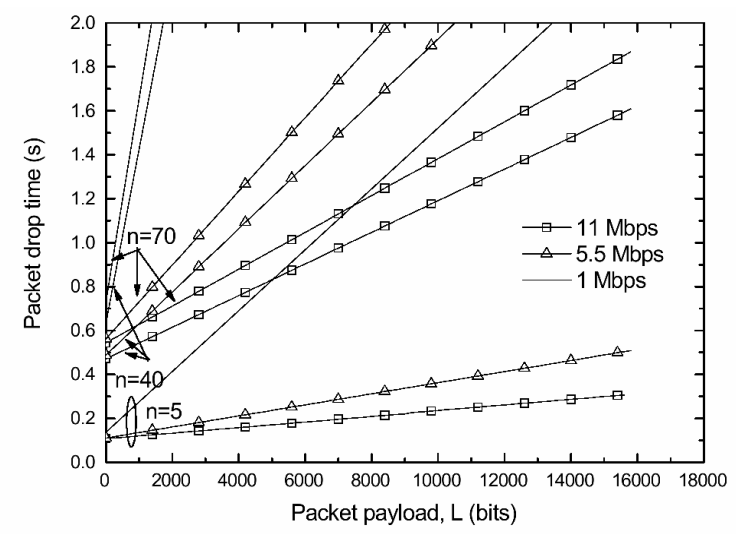

Figure 9. Packet drop time against packet payload length for different WLANs sizes and channel bit rats. 